\title{
EFFECT OF ACTION OBSERVATION THERAPY ON RECOVERY IN UPPER EXTREMITY POST STROKE PATIENTS
}

\section{Keziya Mary Philip, PharmD ${ }^{1}$, Jossy P Jose, PharmD ${ }^{1}$, Shanmuga Sundaram Rajagopal, $\mathrm{PhD}^{2}$, Joice Maria Jose, PharmD ${ }^{2}$}

\#1 Department of Pharmacy Practice, RR College of Pharmacy, Guddadahalli, Bengaluru, Karnataka, India, +91-8681055483, keziyachen93@gmail.com

\#1 Department of Pharmacy Practice, JKK Nattraja College of Pharmacy, Komarapalayam, Tamil Nadu, India, +91-9497014787, drjossyputhumulliljose@gmail.com

\#2 Department of pharmacology, JKK Nattraja College of Pharmacy, Komarapalayam, Tamil Nadu, India, +91-8825873411, malshan34@gmail.com

\#2 Department of Pharmacy Practice, JKK Nattraja College of Pharmacy, Komarapalayam, Tamil Nadu, India, +91-7019527580, joismaria2992@gmail.com

\section{ABSTRACT}

Background: A simple, cost-effective approach for improving upper extremity function, known as action observation therapy by means of exploiting the mirror neurons facilitates motor learning and acts as a novel rehabilitation approach for stroke patients.

Aim: To compare the effectiveness of action observation therapy and conventional drug therapy to improve upper limb function in sub-acute stroke patients.

Methods: A prospective randomized control study in a total of 40 stroke patients was conducted, in which the subjects were divided into 2 groups; those in the control group $(n=20)$ received routine rehabilitation treatment, whereas those in the experimental group $(n=20)$, in addition, received 4 weeks of action observation therapy for 10 minutes/exercise. Experimental group watched videos depicting a model performing specific motor actions typically performed in daily life, before enacting the same actions themselves. All patients were assessed using Fugl-Meyer Assessment and Barthel Index at baseline and at 4 weeks, after treatment. Data were analysed using SPSS 20.0 software.

Findings: After the 4 weeks of treatment, both groups of patients exhibited significant improvement in the measurements $(\mathrm{p}<0.001)$. Moreover, the FMA, BI scores were significantly higher in the experimental group compared to the control group $(p<0.001)$.

Conclusion: AOT appreciably improves upper extremity motor function and performance of activities of daily living in patients with first ever stroke.

Key-words: action observation therapy, mirror neurons, stroke rehabilitation, motor function, daily activities, upper extremity

Corresponding Author: Dr.ShanmugaSundaram Rajagopal, Ph.D

\section{INTRODUCTION}

Stroke is a medical emergency and a leading cause of death worldwide. It occur when the blood vessels supplying the brain burst or more often when a blockage develops. Stroke is one of the leading causes of death and disability in India. The estimated adjusted prevalence 
rate of stroke range 84-262/100,000 in rural and 334-424/100,000 in urban areas. A WHO report says that by the year 2020 , stroke will have moved from the $6^{\text {th }}$ leading cause of lost disability adjusted life years (DALY's) to $4^{\text {th }}{ }^{[1]}$. Motor function loss is common after stroke incidence. The patient may present with severe motor deficits that can limit their activities of daily living. Most commonly the upper extremities and speech are affected.

Recovery of upper extremity function proceeds most rapidly during the initial 3 months after the stroke, but typically slows down after 6 months and reaches a neurological recovery plateau by the end of the first year ${ }^{[2]}$. Upper extremity disorders after stroke can result from weakened or stiff muscles, imbalance, hypertonia and sensory disturbances. Because upper extremity tasks, such as reaching, grasping, manipulating and carrying, require coordination of multiple joints and muscles, recovery of each joint function is necessary before full function is restored. A relatively simple and cost-effective approach for improving upper extremity function, known as action observation therapy, a novel rehabilitation approach exploiting the mirror neurons has shown promise in the treatment of patients with hemiplegia by facilitating motor learning and the building of a motor memory trace in stroke patients ${ }^{[3]}$. Mirror neurons are a distinctive class of neurons discovered in the last decade of neuroscience that modulates their activity both when an individual executes a specific motor act and when they observe the same action performed by another individual $^{[4,5]}$.

A Chinese study showed that the performance of daily living activities, upper extremity function and Ashworth score of stroke patients were improved by action observation therapy, thereby demonstrating the effectiveness of mirror neuron activation on functional recovery after stroke ${ }^{[6]}$. The aim of the present study was to compare the effects of action observation therapy with standard rehabilitation alone on upper extremity function and activities of daily living in hemiplegic patients with stroke.

\section{MATERIALS AND METHODS}

A total of 40 stroke patients who were hospitalized in a tertiary care hospital between January 2016 and June 2016, were recruited for this study. This study was approved by the Ethics Committee (JKKNCP/ETHICS_PRACTICE/0223U16). The criteria for inclusions were: (1) both the genders; (2) patients of age above 45-75 years; (3) patients who are willing to participate and signed in the informed consent letter; (4) patients with unilateral stroke hemiparesis diagnosed and confirmed upon CT and MRI scan; (5) patients with sitting balance $\geq$ level 1; (6) patients with FMA $\geq 10$ for upper extremity motor function and (7) patients with stable condition and who pass the visual imagery questionnaire with normal score. The exclusion criteria include: (1) pregnant and lactating women; (2) pediatric patients; (3) patients with hemorrhagic stroke; (4) patients with co-morbidities that influence voluntary upper-extremity function; (5) psychiatric patients; (6) epileptic patients and (7) patients with liver failure and kidney failure.

Patients eligible for inclusion in the study were divided into experimental and control groups based on a random number table. For the patients in the control group, only the two scales were used to assess the functions but, in the experiment group visual imagery questionnaire and sitting balance were checked prior to the assessment by scales to see if they meet the inclusion criteria.

\section{Research Method}

The Visual Imagery Questionnaire (VIQ) was administered to all patients before and after treatment to determine and assess the specific operation method, procedure, time and intensity of the extremity rehabilitation. The evaluations were carried out by the 
physiotherapist, who had undergone specific professional training, were unaware of the patient grouping status and did not participate in the treatment. Patients in both groups received conventional drug treatment, physiotherapy for a total of four weeks. Patients in the experiment group were made to watch videos of motor activity, which constituted four levels of difficulty namely, level 1: easy, level 2: moderate, level 3: difficult and level 4: very difficult, for 10 minutes per exercise for 04 weeks in experiment groups. Finally, at the time of discharge too, the level of activity was measured using the above mentioned scale for both groups. The scores at baseline and after the course of action observation therapy along with drug therapy were compared in both groups.

For limb movement training, patients were asked to sit at a distance of $2 \mathrm{~m}$ from a laptop set and were required to first watch a video showing a specific action of the upper limb and then perform the same exercise after watching.

A total of 12 action videos were used, which depicted the same model performing the above (Table 1). Videos with similar difficulty levels were grouped into four groups of three videos each; Group 1 videos comprised the easiest actions and Group 4 videos comprised the most difficult. Patients were initially shown videos from Group 1 and instructed to try their best to simulate the action with their affected limbs. Once at least four of the actions could be performed, they moved on to the next group (higher difficulty) of videos.

Table 1: Difficulty level of experiments

\begin{tabular}{|c|c|c|}
\hline Level & Name of Experiments & Time Allotted \\
\hline Easy & $\begin{array}{l}\text { *Sponge exercise } \\
\text { *Finger stretch } \\
\text { *Supination \&Pronation }\end{array}$ & Within 10 seconds \\
\hline Moderate & $\begin{array}{l}\text { *Abduction-Adduction } \\
\text { *Finger to nose } \\
\text { *Hand to hand }\end{array}$ & $15-20$ seconds \\
\hline Difficult & $\begin{array}{l}* \text { PNF } \\
* \text { Grasping of tumbler } \\
* \text { Pray position }\end{array}$ & 1 Minute \\
\hline Very Difficult & $\begin{array}{l}\text { *Dressing } \\
\text { *Grooming } \\
\text { *Feeding }\end{array}$ & 5 Minutes \\
\hline
\end{tabular}

\section{Evaluation Indices}

FMA of upper limb: The motion content of this scale includes the cooperative motions of reflection, shoulder, elbow, wrist and fingers, separation movement and 33 other items; the total integral score is $66^{[4]}$. This scale has good reliability and validity, and is highly recommended for the evaluation of motor function after stroke ${ }^{[6]}$.

BI: The BI was used for evaluating the activities of daily living in the patients with stroke. This scale has good reliability for the evaluation of recovery of function in stroke patients ${ }^{[6]}$.

Statistical Analysis

Data were analysed using SPSS 20.0 software and are expressed as means \pm SD. 


\section{RESULTS}

The data shows no statistically significant difference between the experiment group and control group based on sex, age and affected side (Table 2). There is no significant difference in both groups before treatment. Nevertheless FMA and BI score improved in both control and experimental group after 4 week treatment (Table 3 and 4; $\mathrm{p}<0.05$ ). Moreover, the experimental group shows better response than control group in FMA and BI scores after treatment (Fig.1).

Table 2: Baseline characteristics

\begin{tabular}{|c|c|c|c|c|}
\hline Characteristics & Experimental group & Control group & t value & p value \\
\hline \multicolumn{5}{|c|}{ Sex } \\
\hline Male & 13 & 15 & \multirow{2}{*}{4.000} & \multirow{2}{*}{0.156} \\
\hline Female & 7 & 5 & \\
\hline Age & $58.60 \pm 11.46$ & $59.05 \pm 10.71$ & 0.136 & 0.893 \\
\hline \multicolumn{5}{|c|}{ Affected side } \\
\hline Left & 9 & 12 & 0.000 & \multirow{2}{*}{1.000} \\
\hline Right & 11 & 8 & & \\
\hline
\end{tabular}

Table 3: Scores of FMA and BI in the Experiment Group

\begin{tabular}{|c|c|c|c|c|c|}
\hline Sl. No & $\begin{array}{c}\text { Name of the } \\
\text { Scale }\end{array}$ & $\begin{array}{c}\text { Before } \\
\text { Treatment } \\
(\text { Mean } \pm \text { SD) }\end{array}$ & $\begin{array}{c}\text { After } \\
\text { Treatment } \\
(\text { Mean } \pm \text { SD) }\end{array}$ & t Value & p Value \\
\hline 1. & FMA & $24.20 \pm 5.890$ & $34.35 \pm 5.480$ & -9.513 & $<0.001$ \\
\hline 2. & BI & $20.25 \pm 7.691$ & $5.40 \pm 12.083$ & -14.367 & $<0.001$ \\
\hline
\end{tabular}

FMA- Fugl-meyer assessment BI- Barthel Index

Figure 1: Scores of FMA and Barthel Index in the Experiment Group

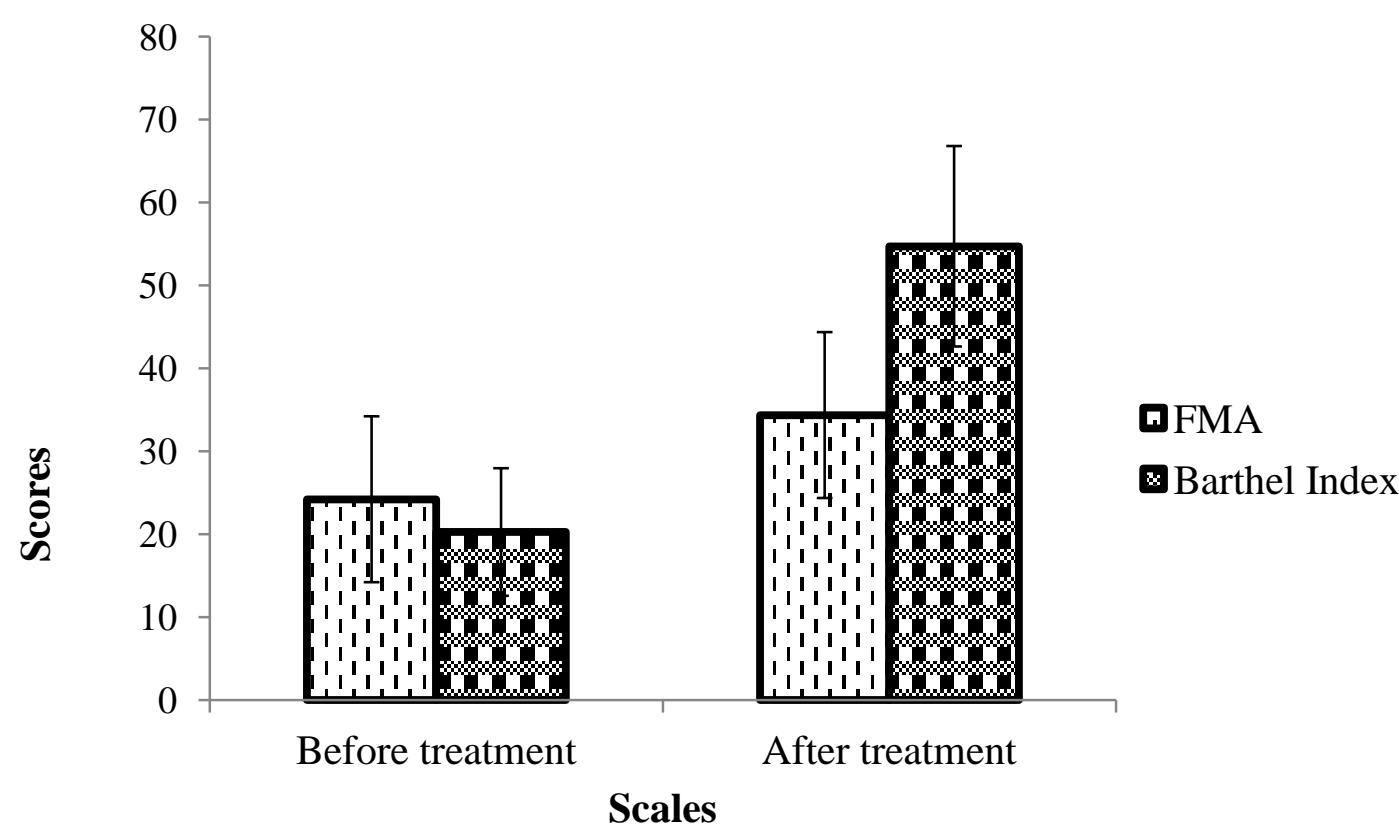


Table 4: Scores of FMA and BI in the Control Group

\begin{tabular}{|c|c|c|c|c|c|}
\hline Sl. No & $\begin{array}{c}\text { Name of the } \\
\text { Scale }\end{array}$ & $\begin{array}{c}\text { Before } \\
\text { Treatment } \\
\text { (Mean } \pm \text { SD) }\end{array}$ & $\begin{array}{c}\text { After } \\
\text { Treatment } \\
\text { (Mean } \pm \text { SD) }\end{array}$ & t Value & p Value \\
\hline 1. & FMA & $21.0 \pm 3.811$ & $24.0 \pm 4.645$ & -6.458 & $<0.001$ \\
\hline 2. & BI & $21.0 \pm 6.907$ & $32.50 \pm 9.801$ & -4.774 & $<0.001$ \\
\hline
\end{tabular}

FMA- Fugl-meyer assessment BI- Barthel Index

Figure 2: Scores of FMA and Barthel index in control group

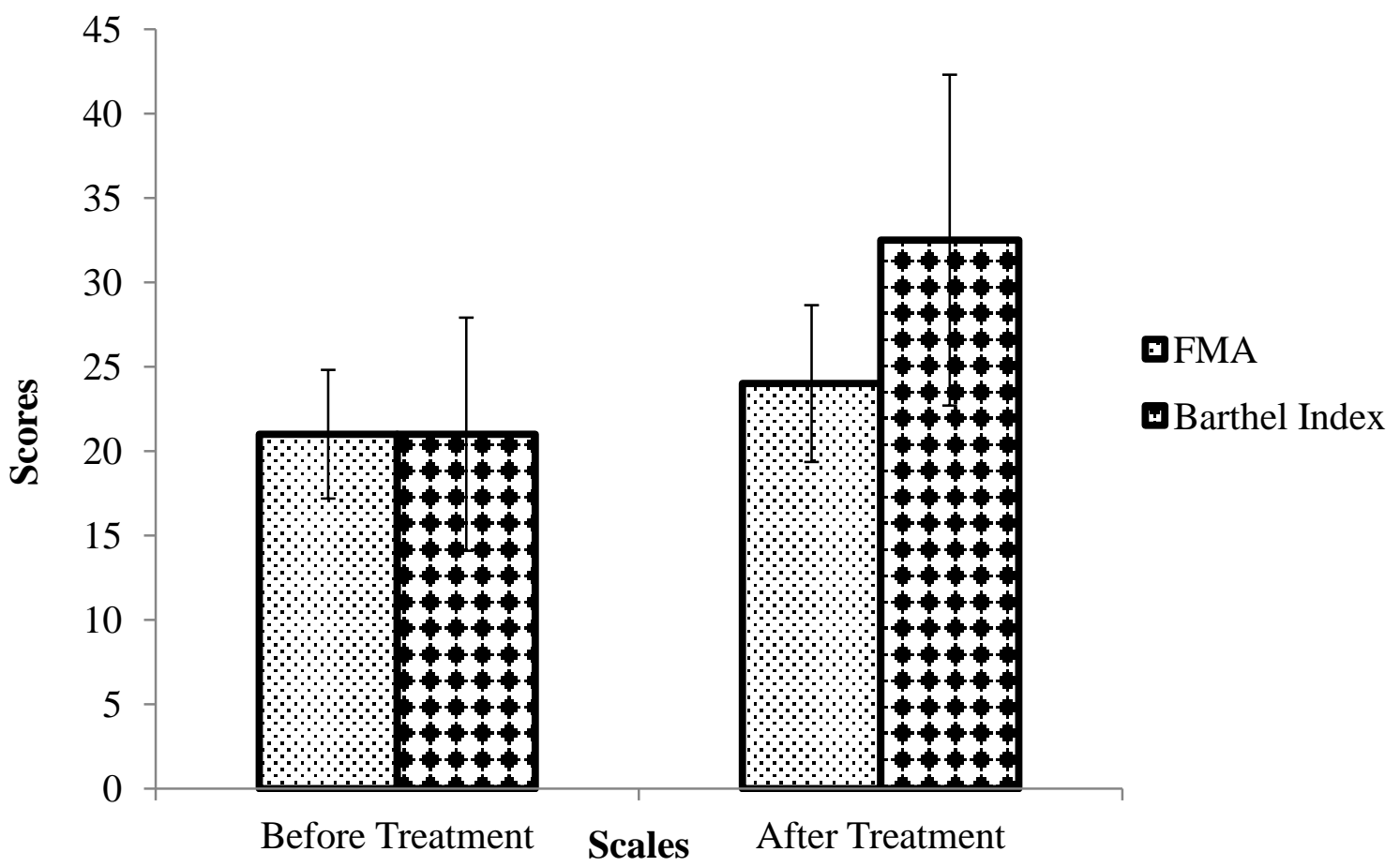

\section{DISCUSSION}

The study was done on 40 patients who were admitted in the hospital 10-15 days before the study. There were no dropouts from the study. The number of male patients was 13 in experimental group and 15 in the control group. The number of female patients was 7 in experimental group and 5 in the control group respectively.

From the present study, there were 9 subjects from the experimental group and 12 subjects from the control group with left side hemiplegia, whereas 11 subjects from the experimental 
group and 8 subjects from the control group affected with right side hemiplegia. The recovery rate was almost the same in both right and left hemiplegic subjects.

The distribution of demographical data shows that there were no statistical difference between the experimental and control group with respect to the sex, age and affected side in the present study. There was no significant difference between the assessment keys (FMA and BI) before therapy among both the groups (FMA Scores- experiment group: $24.20 \pm 5.890$ \& control group: $21 \pm 3.811$; BI Scores- experiment group: $20.25 \pm 7.691$ \& control group: 21+6.907; p-value: 0.75$)$.

Conversely the FMA and BI scores were increased significantly in both groups after the treatment (FMA Scores- experiment group: $34.35 \pm 5.480 \&$ control group: $24 \pm 4.645$; BI Scores- experiment group: $54.70 \pm 12.083 \&$ control group: $32.50 \pm 9.801$; p-value $:<0.001)$. In a similar study, Mei-Hong et al. 2015 shows that patients who underwent action observation therapy along with traditional rehabilitation have superior recovery after eight weeks as assessed by FMA (experiment group: before therapy-27.58 $\pm 9.53 \& 35.52 \pm 12.43$ after therapy), BI (experiment group: before therapy-53.87 $\pm 14.24 \& 75.32 \pm 15.57$ after therapy) and MAS scores, which are reliable and valid assessments. Furthermore, the experiment group which received video therapy in addition to the conventional therapy and drug therapy obtained better scores than the control group which received only conventional therapy along with the drug therapy.

Most of the subjects in our study showed improved upper limb motor function in the first weeks of the therapy. Similar observations were made by Toh and Fong ${ }^{[7]}$ who said Mirror Therapy had improved the upper Limb motor performance of the participants immediately after intervention.

Observation of an action, with the intention to imitate movements, may increase the excitability of the brain motor areas and in doing so, be able to stimulate the recovery of motor control. Eric et al ${ }^{[8]}$ says that the motor imagery creates a visual feedback of the observed action, which leads to activation of mirror neurons in the temporal sulcus of the cerebrum. Our study lacks the support of imaging studies to show the activation of mirror neuron system following action observation ${ }^{[9-13]}$. Our study was in agreement with the other studies which claim that action observation improved motor functioning of upper extremity in stroke patients.

The four weeks hospital based action observation therapy showed noticeable changes in the upper extremity function of post stroke patients. The video therapy was well accepted by the patients because of its simplicity and lack of side effects. This therapy was the first of its kind to be done in our study center. Our study shows that receiving video therapy one hour a day for 4 weeks during hospital stay and 6 weeks follow-up showed a marked increase in the motor functioning of post stroke patients.

\section{CONCLUSION}

The results of this study demonstrates the supplementation of action observation therapy along with conventional rehabilitation therapy and drug therapy improves upper extremity function and daily activities in first ever stroke patients with hemiplegia.

\section{LIMITATIONS}

The current study has some limitations.

- First, because of the limited number of participants and suboptimal randomization procedure, we cannot draw firm conclusions regarding the effectiveness of the experimental intervention.

- The absence of follow-up after the end of the action observation therapy does not allow for determination of the durability of the effect of this therapy. 
- Imaging studies like MRI were not done to confirm the action of mirror neurons.

- Finally, the study does not include diverse evaluation of the walking ability and independent daily routine which are important in quality of life.

Therefore, these results can be generalized to sub-acute stroke patients. Further studies, including a long-term follow-up assessment, are needed to evaluate the long term benefits of mirror therapy. Further studies exploiting MRI to locate cortical stimulation and activation of mirror neurons during action observation therapy would draw a firm conclusion that action observation therapy can be beneficial for neuro-rehabilitation in post stroke patients.

Abbreviations: AOT- Action Observation Therapy, FMA- Fugl-Meyer Assessment, BIBarthel Index, DALY's- Daily activity life years, WHO-World Health Organization, MRIMagnetic Resonance Imaging, CT- Computerized Tomography, PNF- Proprioceptive Neuromuscular Facilitation, VIQ- Visual Imagery Questionnaire

\section{KEY POINTS}

Brain plasticity is most important during the stroke rehabilitation treatment and it's a basis for all functional recovery after stroke. Mirror therapy and action observation therapy exploits the theory of mirror neurons, where the observation or even mind representation of the movement facilitates the activation of the brain areas involved in the performance of the motor tasks. These techniques are generalized, especially for the upper limb, with highly suspected results on motor and functional recovery. The existence of audio visual mirror neurons indicates that auditory access to action representation is present. The audio-visual neurons code only object-related actions. Mirror therapy may be beneficial in improving balance ability among sub-acute stroke patients.

\section{REFERENCES}

1. Jeyaraj D, Paulin S, Stroke Epidemiology and Stroke Care Services in India, $J$ Stroke.,vol.15(3), pp.128-134, 2013.

2. Young-Rim P, Simple and Task-oriented Mirror Therapy for Upper Extremity Function in Stroke Patients: A Pilot Study, Hong Kong J Occup Th., vol.24(8), pp.6-12, 2014.

3. Wade SS, Joey D, Dennis L, Cerebrovascular Diseases, Harrison's Principles of Internal Medicine, eighteenth edition. New York, US: McGraw-Hill, pp.23-73, 2005.

4. Giacomo R, Corrado S, The functional role of the parieto-frontal mirror circuit: interpretations and misinterpretations, Nat Rev Neurosci., vol.11(2), pp.264-274, 2010.

5. Christian D, Violetta N, Thomas H, Klaus S, Mircea A S, Video Therapy: Promoting Hand Function after Stroke by Action Observation Training - a Pilot Randomized Controlled Trial, Int J Phys Med Rehabi.,. vol.2(5), pp.2, 2014.

6. Mei-Hong Z, Jing W, Xu-Dong G, Mei-Fang S, Effect of action observation therapy on daily activities and motor recovery in stroke patients, Int.J.Nat.Soc.Sci., vol.2(3), pp.279282, 2015.

7. Toh SF, Fong KN, Systematic Review on the Effectiveness of Mirror Therapy in Training Upper Limb Hemi paresis after Stroke, Hong Kong J Occup Th., vol.22(2), pp.84-95, 2012.

8. Eric LA, Sidney BW, Lance S, Chris F, Douglas G, Mark E L, Rehabilitation of hemi paresis after stroke with a mirror [Research Letter], Lancet., vol.353(9169), pp.20352036, 1999.

9. Mukamel R, Ekstrom AD, Kaplan J, Iacoboni M, Fried I, Single neuron responses in humans during execution and observation of actions, Curr Biol., vol.20(8), pp.750-756, 2010 . 
10. Maeda F, Kleiner FG, Pascual LP, Motor facilitation while observing hand actions: specificity of the effect and role of Observer's orientation, J Neurophysiol., vol.87(3), pp.1329-1335, 2002.

11. Magda O, Geoffrey B, Cecilia H, Action observation supports effector dependent learning of finger movement sequences, Exp Brain Res., vol.165(2), pp.19-27, 2005.

12. Stefan K, Cohen LG, Duque J, Mazzocchio R, Celnik P, Sawaki L, Formation of a motor memory by action observation. J Neurosci., vol.25(2), pp.9339-9346, 2005.

13. Trempe M, Sabourin M, Rohbanfard H, Proteau L, Observation learning versus physical practice leads to different consolidation outcomes in a movement timing task, Exp Brain Res., vol.209(12), pp.181-192, 2011. 